\title{
Livres
}

\section{L'illusion transsexuelle}

Patricia MERCADER

L'Harmattan, Paris, 1994, 298 pages

Le titre de cet ouvrage synthétise parfaitement les thèses avancées et développées sur la problématique de la demande transsexuelle. L'auteur donne des clefs pour comprendre ce que recouvre la certitude d'un homme se disant "une femme piégée dans un corps d'homme" ou d'une femme se disant "un homme piégé dans un corps de femme." Il n'est pas question de prendre parti pour une origine (biologique, psychologique) du transsexualisme, mais de donner les moyens d'une compréhension sur ces "étranges exceptions" à un caractère fondamental qu'est l'appartenance à UN sexe.

Patricia Mercader introduit sa réflexion par une étude historique sur les différents cas de travestissement, de pratiques sexuelles déviantes comme celle des sodomites brûlés vifs, avant la Révolution. Au XIXème siècle apparaît un discours médical sur les perversions, et plus précisément sur le concept d'inversion. Le début du XXème siècle marque les premières interventions chirurgicales d'hommes et de femmes, repérés par les médecins comme homosexuels, et pour lesquels ce traitement permet de rétablir une identité hétérosexuelle. Ce n'est qu'après la Seconde Guerre que la notion de transsexualisme apparaît. Patricia MerCaDER propose une étude approfondie et une critique des travaux de Harry BENJAMin, John Money et Robert STOLLER ; chacun de ces auteurs essayant d'élaborer une théorie à partir de la biologie, ou des thèses comportementalistes ou de la psychanalyse. Actuellement les théoriciens du transsexualisme proposent des approches différentes : le transexuel est-il border-line, psychotique délirant ou névrosé ? Le diagnostic est difficile tant les ramifications sont nombreuses dans le champ culturel et social. En ligne de fond, se profile toujours la question du traitement hormonal et de l'intervention chirurgicale que certains auteurs refusent et que d'autres proposent.

L'intérêt du livre de Patricia Mercader repose aussi sur son travail de recherche à partir de la jurisprudence des tribunaux français (1965-1993) des demandes de rectification de l'état civil. Ces jugements permettent de voir les fluctuations des décisions à partir de notions comme celle de transsexualisme "vrai", suivant des références biologiques ou psychologiques. L'auteur a aussi analysé les autobiographies publiées par des transsexuels qui s'appliquent à "montrer du vrai" et aussi les souffrances qu'ils endurent. Ces différentes investigations apportent des outils de compréhension provenant de la part de non spécialistes (les magistrats) ou au contraire des sujets eux-mêmes dans le récit de leur cursus.

Le livre de Patricia Mercader propose des éléments de réponse à la question de la demande transsexuelle en incluant l'analyse des représentations sociales les plus construites sur ce sujet. L'auteur ne cherche pas à résoudre le problème de l'identité transsexuelle. On remarque que le syntagme "changement de sexe" est toujours écrit entre guillemets, ce qui tend à prouver que l'auteur ne pense pas qu'un individu puisse changer de sexe et qu'elle n'est pas contaminée par le discours sur la croyance et la conviction qu'une telle entreprise soit possible.

(J.L. CLEMENT) 